\title{
Archaeometallurgical Analysis of Bronze Artifacts: A Magnetometer Approach
}

\author{
Lucas Braddock Chen \\ Foundation for the Advancement of Anthropology \& History, Menlo Park, CA, USA \\ Email: Braddock9955@gmail.com
}

How to cite this paper: Chen, L. B. (2020). Archaeometallurgical Analysis of Bronze Artifacts: A Magnetometer Approach. Archaeological Discovery, 8, 199-206.

https://doi.org/10.4236/ad.2020.83011

Received: April 21, 2020

Accepted: May 12, 2020

Published: May 15, 2020

Copyright $\odot 2020$ by author(s) and Scientific Research Publishing Inc. This work is licensed under the Creative Commons Attribution International License (CC BY 4.0).

http://creativecommons.org/licenses/by/4.0/

\begin{abstract}
Bronze has been utilized by human civilization for the past five millennia, and societies across the globe have mined, extracted, and purified copper and tin to create bronze alloys. Due to the different mineral composition of ores and the varying techniques involved with metal smelting, bronze artifacts from around the world often have very different chemical compositions. The determination of chemical composition is often invasive and expensive, and is usually conducted in laboratories. We previously developed an inexpensive and mobile test to identify metal alloys based on their magnetic signatures. We demonstrated that metals of different compositions would exhibit different electrical conductivity, and thus different magnetic field strengths when evoked by different levels of electric current. In this manuscript, we now detail the experiment protocol to produce evoked dynamic electromagnetic signals from bronze alloys, and the capture of signals with the smartphone magnetometer.
\end{abstract}

\section{Keywords}

Archaeometallurgy, Metallurgy, Bronze, Ancient, Magnetism, Magnetometer

\section{Introduction}

Copper was the first metal widely utilized across the ancient world. Its prevalence, shiny luster, and natural existence as native copper allowed this metal to be noticed and collected. Mesopotamian metalsmiths were able to cold hammer native copper into pins and awls around 6500 - 5500 BC (Gale et al., 1985). However, the supply of native copper was limited. Copper only became widely available after man learned to harvest copper through smelting ores, from which pure copper could be isolated after a series of heating and reduction-oxidation reactions using forced air and charcoal (Muhly, 1985). Copper, however, was soft and had limited durability as tools and weapons (Curry, 2020). In addition, 
its high melting temperature of 1084-degrees Celsius also rendered the metal laborious to cast using a conventional open fire pit (Bennett, 2020). The development of bronze thus represented a major technological advance in $3300 \mathrm{BC}$ when mankind discovered that the tin and copper alloy is stronger, more resistant to corrosion, and more malleable (Lopez, 2009; Raymond, 1984).

These ancient bronzewares, however, were often of very different chemical composition and properties, as they were smelted from distinct copper ores containing varying minerals, depending on the geology of the ore origin. In addition, copper is often composed of different molecules, such as oxides, carbonate, and sulphide compounds (Muhly, 1976). Copper oxides include cuprite $\mathrm{Cu}_{2} \mathrm{O}$ and melaconite $\mathrm{CuO}$; copper carbonate includes malachite $\mathrm{Cu}_{2}(\mathrm{OH})_{2} \mathrm{CO}_{3}$ and azurite $\mathrm{Cu}_{3}(\mathrm{OH})_{2}(\mathrm{CO} 3)_{2}$; and copper sulfide includes chalcocite $\mathrm{Cu}_{2} \mathrm{~S}$ and covellite CuS. Copper isolated from these compounds have different levels of purity, and the associated minerals were invariably also extracted during the smelting process: this resulted in unique chemical signatures for the smelted copper. Similarly, tin extracted from ores were also of unpredictable purity levels, containing minerals based on the ore geology. Consequently, when bronze was created, the mixture of copper and tin invariably created a final alloy of unique chemical signatures.

As a result, the identification and classification of ancient bronze artifacts can be challenging, as artifacts of similar shapes and appearance may nevertheless consist of completely different chemical compositions, and thus, chemical signatures. It nevertheless presents an opportunity to uniquely identify each bronze artifact and possibly understand its history and origin. The study of archaeometallurgy has employed various techniques in an attempt to better characterize bronzeware. Several different noninvasive tests have been developed. For example, a streak test can assess metal hardness by scratching the surface, but this is an unreliable method to identify metal content. Energy spectrometry projects an $\mathrm{X}$-ray beam to the metal's surface and examines the emitted secondary $\mathrm{x}$-ray signal, which can help provide characteristics of the metal. However, this technique can only detect the metal on the surface and is not usable if the antique is painted or plated with a different metal (Raymond, 1984).

Some invasive methods have also been utilized. A spot analysis dissolves a small piece of metal in a chemical mix to determine metal composition, but the process is destructive and can only sample a small portion of the metal artifact. Neutron activation analysis places a metal sample in a nuclear reactor and examines the emitted gamma-ray from bombarding neutron beams. This metal identification process, however, is expensive, destructive, and can only analyze a small sample. Moreover, it has been shown that copper and tin often do not mix uniformly as they have different melting points; thus, bronze artifacts have different metal compositions throughout their structure (Logan, 2007). Consequently, taking a small sample of the bronze for atomic or chemical analysis may provide misleading information (Scott, 1991). Taking larger or more samples, while providing more data, would severely damage or destroy the valuable artifact. 
None of the above methods of bronze identification are ideal, and some of the techniques require expensive laboratories. As a result, these methods are not readily available nor practical for archaeologists examining an excavated bronze artifact at a dig site. We now report a noninvasive and mobile method of rapid bronze analysis using the magnetometer on a smartphone to determine dynamic magnetic property.

We previously patented a rapid method of steel blade identification based on magnetic properties (Chen, 2019). Steel blades of different grades and qualities are constructed of different ratios of iron, nickel, carbon, and other elements; thus, each blade grade has its own electric conductivity pattern. Since a magnetic field is generated while the blade conducts electricity, each blade grade will have its own induced magnetic field property. Moreover, since magnetic properties can change at different levels of electrical current, each blade class will demonstrate a unique magnetic profile at different levels of electricity (dynamic magnetic property). By measuring the dynamic magnetic profile at different electric conduction levels, one can readily identify the composition of the blade.

Furthermore, we previously reported that the magnetic field can be readily detected using a smartphone (Chen, 2019). All modern smartphones possess a magnetometer as part of their internal compass (Baldini, 2017; Odenwald, 2019), and these magnetometers can be utilized to capture the blade's magnetic profile-both the static magnetic profile (before electricity is applied) and the dynamic profile (determined at different levels of electricity; Arribas, 2015). The protocol of identifying steel composition using a smartphone magnetometer was recently published (Chen, 2020a). In addition, we showed that the dynamic magnetic signatures corresponded to the chemical composition of the steel blade and its Vickers microhardness (Vickers Hardness Test, 2020; Chen, 2020b).

We recently patented a method of identifying bronze artifacts using a similar technology (Chen, 2020c). As stated above, bronzeware from each region and civilization is composed of different levels of copper and tin since the local metal ores are different. Since copper and tin have different electrical conductivity (with tin only having 15\% of copper's electrical conductivity; Metal Supermarkets, 2015), bronzeware of different copper-tin ratios will have different electrical conductivities, and thus, different dynamic electromagnetic profiles. In addition, as much of ancient bronzeware invariably contains various combinations of arsenic, lead, phosphorus, aluminum, manganese and silicon from local ores, bronze made in different parts of the world have very different magnetic profiles. In this article we now present the experimental protocol necessary for obtaining electromagnetic signatures of bronze artifacts using a smartphone magnetometer.

\section{Material and Methods}

A smartphone can be used to scan bronzeware and detect the dynamic magnetic profile at different levels of electric current to construct its unique magnetic signature. The protocol for magnetic signature extraction requires the following 
parts: a test material holder, a smartphone with a magnetometer, a stabilizing platform for the smartphone, an app for measuring electromagnetic profiles, a source of electricity, and wires to conduct electricity from the electric source to the test material.

1) Test material platform: A holder is used to stabilize the test material during the test. The holder needs to be constructed of electrical insulating material so as not to interfere with the electromagnetic measurement during the test. Small artifacts can be held by the Everbilt Small 2-7/8 in. Rubber Grip Wall Mount Holder (Home Depot, Atlanta, GA). As for more delicate materials, the Web clamp is used (DeWalt, Baltimore, MD). Heavy objects would be better stabilized by the robust plastics jaw clamp (Home Depot, Atlanta, GA). For the current study, the Multifunctional Welding LED Magnifier Helping Hand Soldering Iron Stand (Amazon, Seattle, WA) was used (Figure 1).

2) Smartphone with magnetometer and software: Most smartphones now contain a magnetometer as a part of their internal compass. An iPhone XS Max, Apple (Cupertino, CA) running iOS 12.4 was used in the current study. Magnetscape 2.0 (Toon, Osaka, Japan) was used as the magnetometer software to record electromagnetic signatures.

3) Smartphone stabilizer. A rubber-insulated holder was used to hold and stabilize the smartphone during the study. For the iPhone used in the study, Aduro Solid-Grip Phone Holder for Desk-Adjustable Universal Gooseneck Smartphone Stand, with Durable Rubberized Mount, was used as the stabilizer (Amazon, Seattle, WA).

4) Electric source: In order to obtain magnetic signatures at different energy levels, a variable energy source was used: Tekpower TP3016M Portable Handheld Variable DC Power Supply with USB Port, 0.3 V - 12 V @ 0 - 3.75 A or 0.3 V-30 V@1.6 A with VC and CC Control, Upgraded TP3005D, HY3005 (Tekpower, Montclair, CA; Figure 2(a)).

5) Resistor. In order to accommodate the variable currents needed for the extraction of magnetic signatures, a Resistance Substitution Box Model RS-400 (Elenco Electronics, Wheeling, IL) was used (Figure 2(b)).

6) Connecting cables. Alligator Clip with Pigtail (Amazon, Seattle, WA) connecting cables was used to establish the circuit with the test material.

7) Standardization copper and bronze disks. In order to obtain baseline electromagnetic signatures, standardization copper and bronze metal disks were used: Metal 1" Disc Sample Pack: bronze, copper (K \& S Engineering, Highland IN; Figure 2(c)).

\section{Results}

Copper and bronze metal disks were mounted onto the platform and the smartphone was stabilized above the specimen for magnetic field detection. Measurements were taken to acquire baseline magnetic signatures. Electric charges were then applied to the metal disks at 6 volts to induce an electromagnetic field (Figure 3). 
Electromagnetic signatures were measured in MicroTesla $(\mu \mathrm{T})$, with results recorded in Table 1. Five different measurements were taken at each point and the averages were presented. At baseline, the copper disk demonstrated 33.26 $\mu \mathrm{T}$, while bronze (which contains significant amounts of tin) demonstrated $32.15 \mu \mathrm{T}$. When both disks were placed in the 6-volt electric circuit, the evoked dynamic electromagnetic strength of copper was $34.06 \mu \mathrm{T}$, a net increase of 0.8 $\mu \mathrm{T}$. The evoked electromagnetic strength of bronze was $32.62 \mu \mathrm{T}$ with the input of 6-volt electric circuit, with a net increase of $0.47 \mu \mathrm{T}$.

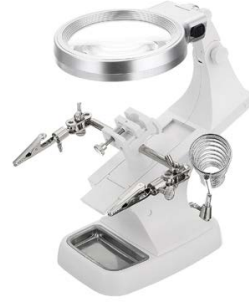

(a)

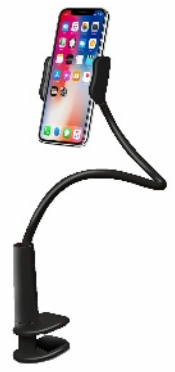

(b)

Figure 1. Experimental platform: (a) Multifunctional Welding LED Magnifier Helping Hand Soldering Iron Stand was used as the stabilizing platform for the test material; (b) Aduro Solid-Grip Phone Holder for Desk-Adjustable Universal Gooseneck Smartphone Stand, with Durable Rubberized Mount, was used as the smartphone holder.

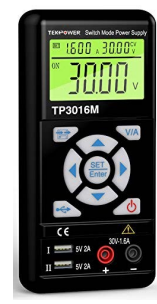

(a)

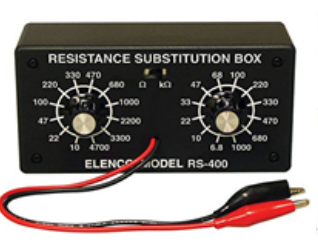

(b)

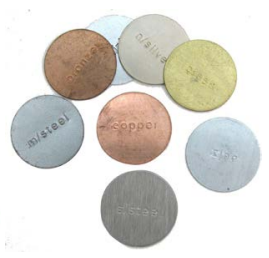

(c)

Figure 2. Variable power source and variable resistor: (a) Tekpower TP3016M Portable Handheld Variable DC Power Supply with USB Port, 0.3 V - 12 V @ 0 - 3.75 A or $0.3 \mathrm{~V}-30 \mathrm{~V} @ 1.6 \mathrm{~A}$ with VC and CC Control, Upgraded TP3005D, HY3005; (b) Resistance Substitution Box Model RS-400; (c) Calibration metal disks (Metal 1" Disc Sample Pack: bronze, copper).

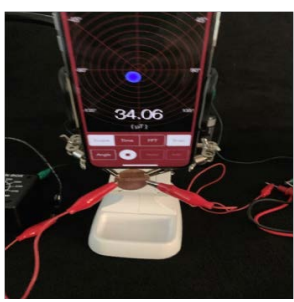

(a)

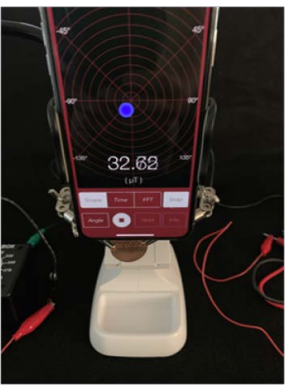

(b)

Figure 3. Magnetic field measurements for (a) copper and (b) bronze at $6 \mathrm{~V}$. 
Table 1. Electromagnetic field measurements of copper and bronze at baseline and in dynamic 6-volt circuit.

\begin{tabular}{ccc}
\hline & Copper & Bronze \\
\hline Magnetism in MicroTesla $(\mu T)$ & & \\
Baseline Magnetism & 33.26 & 32.15 \\
Electromagnetism & 34.06 & 32.62 \\
$\quad$ Differential & 0.8 & 0.47 \\
Magnetic Signatures & & \\
\hline
\end{tabular}

\section{Discussion}

Bronze artifacts are valued for their luster and durability and have been continuously produced across the world since the dawn of the Bronze Age in 3300 BC. Copper and tin ores were harvested from different mines across the world, and their local geography often dictated the types of minerals that were commingled with copper and tin as they were purified and extracted. In addition, since these ancient alloys were forged before the creation of the Periodic Table and knowledge of chemical reactivities and properties, there was considerable variability as to the purity and relative ratio of different elements within each bronze artifact. Bronze alloys created via such techniques would thus have unique chemical signatures and may provide a method to uniquely identify bronze produced from different regions of the world.

The ability to chemically analyze bronze is often expensive and destructive and invariably involves expensive equipment in large laboratories. We previously developed a technique to identify different steel alloys based on their differing magnetic signatures due to different chemical compositions (Chen, 2019), and we demonstrated that the technique can be used also to analyze bronze alloys (Vickers Hardness Test, 2020). In the current article we detail the experimental protocol for conducting such an extraction of dynamic magnetic signatures.

The current technique is possible due to the wide availability of smartphones, which provide a portable and inexpensive yet powerful magnetometer. In the current study we observed different electromagnetic measurements and differentials between copper and bronze specimens. The higher electromagnetic level observed with copper was most likely due to the higher electroconductivity of copper versus bronze. In addition, copper not only showed a higher electromagnetic field at baseline, it also showed a higher spike of evoked electromagnetic level when stimulated by an electric circuit, as compared to bronze.

In conclusion, there had not been a simple, inexpensive, and portable method of assessing the metal composition of an alloy and, indirectly, its identity. The current method of utilizing dynamic electrodynamic signatures as a method of differentiating copper and bronze artifacts represents a novel method to identify bronze objects of different compositions. The next step of research would need to establish a standardization table of electromagnetic signatures of bronze alloys containing different copper-to-tin ratios. In addition, such a table would need to 
be repeated at different electric currents in order to document the evoked dynamic electromagnetic signatures. Having such a database of measurements would help researchers compare any new bronze artifact to the set of known electromagnetic signatures, and may help to estimate the chemical composition of the query bronze material.

\section{Conflicts of Interest}

The author declares no conflicts of interest regarding the publication of this paper.

\section{References}

Arribas, E. (2015). Measurement of the Magnetic Field of Small Magnets with a Smartphone: A Very Economical Laboratory Practice for Introductory Physics Courses. European Journal of Physics, 36, 11. https://doi.org/10.1088/0143-0807/36/6/065002

Baldini, G. B. (2017). Identification of Mobile Phones Using the Built-In Magnetometers Stimulated by Motion Patterns. Sensors, 17, 783. https://doi.org/10.3390/s17040783

Bennett, D. R. (2020). Stages of Wood Burning Combustion. http://www.flameandcomfort.com/archives/blog/311

Chen, L. (2020a). Differentiation of Fencing Blade Alloys Using iPhone Magnetometer. Journal of Experimental Techniques and Instrumentation. (In Press)

Chen, L. B. (2019). Determining Fencing Blade Quality Using Dynamic Magnetic Field Measurements. United States Patent and Trademark Office.

Chen, L. B. (2020b). Rapid Identification of Saber Steel Flexibility, Microhardness, and Chemical Composition by Analyzing Electromagnetic Signatures. American Journal of Sports Science, 8, 29-32.

Chen, L. B. (2020c). Determining Composition of Metal Artifacts Using Dynamic Electromagnetic Profile Managements. United States Patent and Trademark Office.

Curry, A. (2020). Sword-Wielding Scientists Show How Ancient Fighting Techniques Spread across Bronze Age Europe. Science News. https://doi.org/10.1126/science.abc2715

Gale, N. H., Stos-Gale, Z. A., \& Gilmore, G. R. (1985). Alloy Types and Copper Sources of Anatolian Copper Alloy Artifacts. Anatolian Studies, 35, 143-173.

https://www.jstor.org/stable/3642880 https://doi.org/10.2307/3642880

Logan, J. (2007). Identifying Archaeological Metal-Canadian Conservation Institute (CCI) Notes 4/1. Government of Canada.

https://www.canada.ca/en/conservation-institute/services/conservation-preservation-publ ications/canadian-conservation-institute-notes/identifying-archaeological-metal.html

Lopez, A. M. (2009). Metalworking through History: An Encyclopedia. Westport, CT: Greenwood.

Metal Supermarkets (2015). Which Metals Conduct Electricity? https://www.metalsupermarkets.com/which-metals-conduct-electricity/

Muhly, J. D. (1976). Copper and Tin: The Distribution of Mineral Resources and the Nature of the Metal Trade in the Bronze Age. Hamden: Archon.

Muhly, J. D. (1985). Sources of Tin and the Beginnings of Bronze Metallurgy. American Journal of Archaeology, 89, 275-291. https://www.jstor.org/stable/504330 
https://doi.org/10.2307/504330

Odenwald, S. (2019). Smartphone Sensors for Citizen Science Applications: Radioactivity and Magnetism. Citizen Science: Theory and Practice, 4, 18. https://doi.org/10.5334/cstp.158

Raymond, R. (1984). Out of the Fiery Furnace: The Impact of Metals on the History of Mankind. University Park, PA: Penn State University.

Scott, D. A. (1991). Metallography and Microstructure of Ancient and Historic Metals. Marina del Rey, CA: Getty Conservation Institute in Association with Archetype Books. http://hdl.handle.net/10020/gci_pubs/metallography_microstructure

Vickers Hardness Test (2020). https://www.gordonengland.co.uk/hardness/vickers.htm 